Agron. Mesoam. 30(1):165-178. Enero-abril, 2019

ISSN 2215-3608, doi:10.15517/am.v30i1.34094

http://www.revistas.ucr.ac.cr/index.php/agromeso

\title{
Adaptación de diez cultivares de Lolium sp. en el trópico alto de Nariño, Colombia ${ }^{1}$
}

\section{Adaptation of ten cultivars of Lolium sp. in the high tropic of Nariño, Colombia}

\author{
Máryory Maricela Cadena-Guerrero², Mario Augusto García-Dávila ${ }^{3}$, Diego Hernán Meneses-Buitrago², \\ Sonia Patricia Morales-Montero ${ }^{2}$,Edwin Castro-Rincón ${ }^{2}$
}

1 Recibido: 23 de julio, 2018. Aceptado: 9 de setiembre, 2018. Este trabajo formó parte de un proyecto de tesis de maestría llevado a cabo en la Corporación Colombiana de Investigación Agropecuaria (AGROSAVIA), con recursos del Sistema General de Regalías. Colombia.

2 Corporación Colombiana de Investigación Agropecuaria (AGROSAVIA), Centro de Investigación Obonuco, Pasto, Colombia. mmcadena@ agrosavia.co, dmeneses@agrosavia.co (https://orcid.org/0000-0003-3033-3079), spmorales@agrosavia.co, ecastro@agrosavia.co (https:// orcid.org/0000-0001-9841-8242).

3 Universidad Nacional de Colombia, Palmira, Colombia. magarciada@unal.edu.co

\section{Resumen}

Introducción. Los sistemas de producción de leche en el departamento de Nariño, Colombia, se caracterizan por la predominancia del pasto kikuyo (Cenchrus clandestinus), el cual presenta susceptibilidad a heladas, plagas y patógenos, lo que reduce su productividad. Objetivo. El objetivo de este trabajo fue evaluar la adaptación de diez genotipos de raigrás en tres localidades de la zona alta de Nariño (2905-3157 msnm) durante época seca y lluviosa. Materiales y métodos. El estudio se desarrolló de febrero de 2016 a febrero 2017. Se usó un diseño de bloques completos al azar con arreglo multifactorial, y los factores genotipos (diez), localidad, época (seca y lluviosa) y edad de cosecha $(25,30,35$ y 40 días). Se midió la altura de planta, la cobertura, la severidad de enfermedades y el rendimiento de materia seca. Resultados. La respuesta de los genotipos estuvo afectadada por la época. En la localidad de Pasto, en época lluviosa, los valores más altos para altura de planta, cobertura y rendimiento de materia seca se obtuvieron con los genotipos Conquistador (46,33 cm), Aubade (99,67\%) y Tetralite II, Bestfor Plus, Aubade y Boxer (2,22-2,09 t/ha/corte), respectivamente; la severidad de enfermedades más baja en esta localidad la presentó Samson $(1,14)$. Para las mismas variables en Cumbal, en época lluviosa, se destacaron Bestfor $(32,60 \mathrm{~cm})$, Aubade $(90,62 \%)$, Bestfor y Tetralite II $(1,60 \mathrm{t} / \mathrm{ha} /$ corte $)$ y Boxer $(1,06)$; y en Sapuyes fueron Bestfor $(25,33 \mathrm{~cm})$, Boxer $(98,53 \%)$, Bestfor y Tetralite II $(0,66$ y 0,65 t/ha/corte) y Samson, Conquistador y Angus (con valores de 1,0). Conclusión. La época lluviosa afectó positivamente la respuesta de los genotipos con mayores rendimientos de materia seca, altura de planta y cobertura. La respuesta de los genotipos se afectó por las épocas seca y lluviosa, se afectó el rendimiento de materia seca, altura de planta y cobertura. Los genotipos con buen comportamiento agronómico se sugieren para validación comercial para diversificar la oferta forrajera en las localidades estudiadas.

Palabras clave: raigrás, genotipos, adaptación, forraje, materia seca.

\begin{abstract}
Introduction. Dairy systems at the Nariño department in Colombia, are characterized by the presence of kikuyo grass (Cenchrus clandestinus), which is susceptible to frost, pests and patogens reducing biomass productivity.
\end{abstract}


Objective. The objective of this research was to evaluate the adaptation of ten genotypes of ryegrass in three localities at the high lands (2905 - 3157 masl) and to determine the behavior in dry and rainy season. Materials and methods. The study was carried out from February 2016 to February 2017. A complete randomized block designwith four repetitions was used with a multifactorial arrange including genotypes (ten), locality, season (dry or rainy) and time of harvesting $(25,30,35$ and 40 days). Plant height, coverage, severity of diseases and yield of dry matter were measured. Results.There was a response of genotypes to dry and rainy season, improving height, cover and yield of dry matter in rainy season. The genotypes with highest values for plant height, cover percentage, yield of dry matter and the lowest severity of disease at Pasto locality in rainy season were Conquistador (46,33 cm), Aubade $(99,67 \%)$, Tetralite II, Bestfor Plus, Aubade and Boxer (2,22-2,09 t/ha/cut), and Samson $(1,14)$. In the same order of variables in rainy season at Cumbal locality, were genotypes Bestfort $(32,60 \mathrm{~cm})$, Aubade $(90,62 \%)$, Bestfor and Tetralite II $(1,60 \mathrm{t} / \mathrm{ha} / \mathrm{cut})$, and genotype Boxer $(1,06)$; and for Sapuyes locality were Bestfor $(25,33 \mathrm{~cm})$, Boxer $(98,53 \%)$, Bestfor and Tetralite II $(0,66$ y 0,65 t/ha/cut $)$, and Samson, Conquistador and Angus genotypes $(1,0)$ with the lowest severity of diseases. Conclusion. The rainy season affected the response of the genotypes, with higher yield of dry matter, plant height and cover. The response of the genotypes was affected by the dry and rainy seasons, the yield of the dry mater, the plant height and coverage were affected.The genotypes with good agronomic behavior are suggested for commercial validation to diversify the forage supply in the localities studied.

Keywords: Ryegrass, genotypes, adaptation, forage, dry matter.

\section{Introducción}

El sector ganadero es responsable de la mayor parte del uso mundial de tierras, en donde la superficie total de tierra ocupada por pastos equivale al 26\% de la superficie libre de hielo (FAO, 2018). Colombia se ubica como el cuarto productor de leche de América Latina después de México, Brasil y Argentina. El sector lácteo en este país es sumamente importante para la economía nacional, ha participado en la última década con el $2 \%$ del PIB nacional y el 24,3\% del PIB agropecuario, representa cerca de 350000 productores a nivel nacional y genera más de 700 000 empleos directos (MADR y MinCIT, 2016). La producción lechera hace presencia en veintidós departamentos, siendo Antioquia, Boyacá, Cundinamarca y Nariño, las cuatro cuencas lecheras del trópico alto donde más leche se produce (Pinto, 2017).

La cuenca lechera de Nariño está conformada por los municipios de Pupiales, Ipiales, Aldana, Carlosama, Guachucal, Cumbal, Túquerres, Sapuyes, Pasto, Tangua, Yacuanquer y Buesaco. Para el año 2014, entre estos municipios se logró un volumen diario de 820000 litros, que representaron el 5\% de la producción nacional (Castrillón, 2014). De la ganadería de leche especializada en el trópico alto de Nariño dependen alrededor de 31474 productores y 115 empresas asociativas e individuales, quienes deben superar limitantes relacionadas con la variabilidad y estacionalidad de la oferta de forraje, constituida en gran medida por el pasto kikuyo (Cenchrus clandestinus) (AGROSAVIA, 2016). Este forraje naturalizado es susceptible a heladas, plagas y patógenos (Castro et al., 2009; Cárdenas, 2008), que afectan su productividad, lo cual se traduce en mermas productivas de leche, inestabilidad en la producción; además, la rentabilidad se reduce por los gastos en que se incurre por el uso de suplementos concentrados de alto costo (AGROSAVIA, 2016).

La introducción y selección de especies forrajeras con alto potencial de producción, calidad, persistencia y adaptación a las condiciones climáticas y edáficas del trópico alto, es una de las formas más efectivas para incrementar y mejorar la producción y productividad del sector lechero. Estudios realizados en especies forrajeras han evaluado la adaptación de varios genotipos de raigrás (Lolium sp.) al medio, para mejorar la competitividad de los sistemas de producción de leche del trópico alto colombiano, reportando buenos resultados en producción de materia seca y calidad nutricional (Cuesta, 2008; Posada et al., 2013). 
Según el Consolidado Agropecuario de Nariño (Secretaria de Agricultura y Desarrollo Rural de Nariño y Universidad Sergio Arboleda, 2016), en el trópico alto de este departamento existen 160128 ha dedicadas a pastos, donde el 80\% del área (128 102 ha) está en praderas naturalizadas de kikuyo y el 20\% restante (32025 ha) corresponde a otros forrajes naturalizados, de los cuales un alto porcentaje son de raigrás. Sin embargo, existe poco conocimiento sobre el comportamiento de los cultivares que manejan los productores, quienes hacen uso de la oferta de los almacenes, la cual en ocasiones no satisface sus necesidades. Por lo tanto, es importante evaluar distintos genotipos de raigrás que contribuyan a mejorar la oferta forrajera. La presente investigación tuvo como objetivo evaluar la adaptación de diez genotipos de raigrás en tres localidades del trópico alto de Nariño (2905 $3157 \mathrm{msnm}$ ) y determinar su comportamiento en época seca y lluviosa.

\section{Materiales y métodos}

\section{Localización}

En el periodo comprendido entre febrero de 2016 y febrero de 2017, se establecieron tres experimentos en los municipios de Pasto, Cumbal y Sapuyes, con parámetros agroclimáticos promedio indicados en el Cuadro 1.

Cuadro 1. Ubicación de los experimentos para evaluar la adaptación de diez genotipos de raigrás (Lolium sp.) en época seca y lluviosa, y parámetros agroclimáticos de las localidades de Nariño, Colombia. 2016-2017.

Table 1. Experiments localities to evaluate the adaptation of ten ryegrass (Lolium sp.) genotypes in dry and rainy seasons, and agro-climatic parameters at the Nariño, Colombia. 2016 -2017.

\begin{tabular}{|c|c|c|c|c|c|c|}
\hline Municipio & Localidades & Coordenadas & $\begin{array}{l}\text { Altitud } \\
\text { (msnm) }\end{array}$ & $\begin{array}{l}\text { Temperatura } \\
\text { media anual } \\
\left({ }^{\circ} \mathrm{C}\right)^{1}\end{array}$ & $\begin{array}{l}\text { Precipitación } \\
\text { media anual } \\
\quad(\mathrm{mm})^{1}\end{array}$ & Textura de suelo \\
\hline Pasto & $\begin{array}{l}\text { Centro de investigación } \\
\text { Obonuco }\end{array}$ & W $77^{\circ} 10^{\prime} 010^{\prime \prime}$ & 2905 & 13,8 & 1273 & Franco arenosos \\
\hline Cumbal & $\begin{array}{l}\text { Vereda San José } \\
\text { Finca de productor }\end{array}$ & $\begin{array}{l}\text { N } 00^{\circ} 53^{\prime} 12,1^{\prime \prime} \\
\text { W } 77^{\circ} 48^{\prime} 8,06^{\prime \prime}\end{array}$ & 3210 & 10,5 & 1034 & Franco arcillosos \\
\hline Sapuyes & $\begin{array}{l}\text { Granja Exp. Chimangual } \\
\text { Universidad de Nariño }\end{array}$ & $\begin{array}{l}\text { N } 01^{\circ} 02^{\prime} 6,55^{\prime \prime} \\
\text { W } 77^{\circ} 45^{\prime} 3,88^{\prime \prime}\end{array}$ & 3157 & 11,2 & 1061 & Franco areno - gravilloso \\
\hline
\end{tabular}

Fuente/Source: ${ }^{1}$ Climate-Data.org (2018).

* Corporación Colombiana de Investigación Agropecuaria.

\section{Materiales evaluados}

Se evaluaron diez genotipos de raigrás correspondiente a dos variedades anuales (Magnum y Angus [Lolium multiflorum]), dos variedades perennes (Ohau y Samson [Lolium perenne]) y seis híbridos (Bóxer, Aubade, Bestfor Plus, Tetralite II, Bison y Conquistador [Lolium hybridum]). 


\section{Diseño experimental}

Los experimentos se establecieron bajo un diseño de bloques completos al azar (DBCA), con arreglo factorial $3 * 2 * 4 * 10$, con cuatro repeticiones y diez tratamientos (genotipos de raigrás). Cada unidad experimental tuvo un área de $6 \mathrm{~m}^{2}$.

El análisis de la información se realizó con un modelo estadístico multifactorial, considerando como factores los genotipos, la localidad (3), la época de cosecha (seca y lluviosa) y la edad de cosecha (25, 30, 35 y 40 días). Se realizó un procedimiento GLM y un análisis de varianza combinado, donde se evaluaron el efecto principal de cada factor y sus interacciones. Cuando se presentaron diferencias estadísticas significativas se hicieron comparaciones múltiples de promedios de Duncan $(0,05)$ de las variables dependientes: altura de planta, porcentaje de cobertura, severidad de enfermedades y rendimiento de materia seca, utilizando el software SAS (V 9.4).

\section{Variables evaluadas}

\section{Altura de planta $(\mathrm{cm})$}

Antes de cada uno de los muestreos de forraje, se midió la altura de cinco plantas, seleccionadas al azar en cada unidad experimental y haciendo uso de un marco de muestreo de $1 \mathrm{~m}^{2}$. La altura se midió en centímetros desde el suelo hasta el punto más alto de la planta sin estirarla y sin cortar la inflorescencia.

\section{Cobertura $(\%)$}

Antes de cada corte, se evaluó la cobertura de los pastos en porcentaje, del área delimitada por el marco de muestreo de $1 \mathrm{~m}^{2} \mathrm{y}$ con base en la siguiente escala (Figura 1).

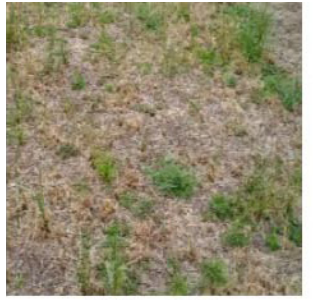

$0(0-9 \%)$

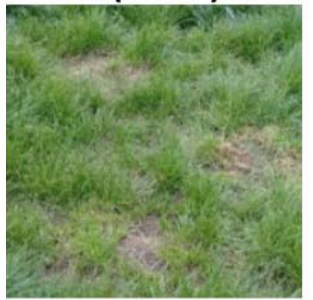

$5(51-60 \%)$

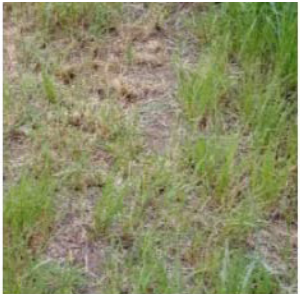

$1(10-20 \%)$

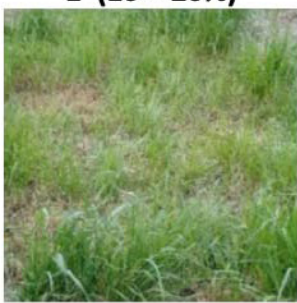

$6(61-70 \%)$

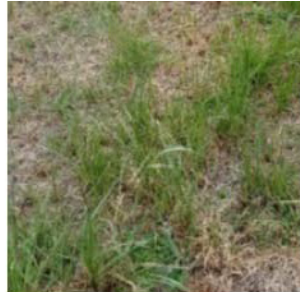

$2(21-30 \%)$

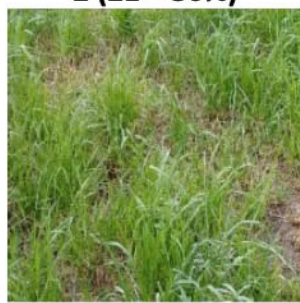

$7(71-80 \%)$

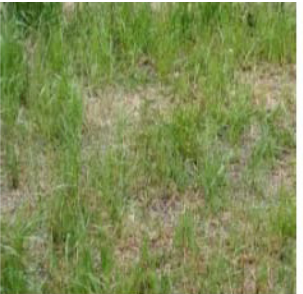

$3(31-40 \%)$

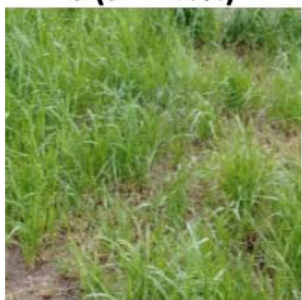

$8(81-90 \%)$

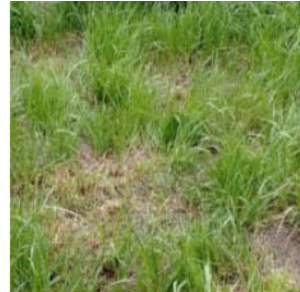

$4(41-50 \%)$

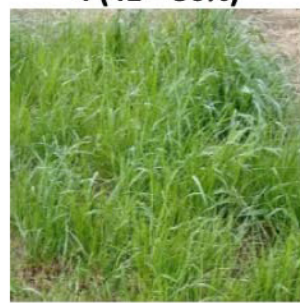

$9(91-100 \%)$

Figura 1. Escala (0-9) para estimar el porcentaje de cobertura en raigrás (Lolium sp.). Nariño, Colombia. 2016-2017.

Figure 1. Scale (0-9) for estimation of the coverage percentage in Ryegrass (Lolium sp.) swards. Nariño, Colombia. $2016-2017$. 


\section{Enfermedades}

Antes de cada corte, se evaluó la severidad de las manchas foliares (Pyricularia grisea) bajo condición de inóculo natural, sin determinar la presencia de razas en plantas afectadas presentes dentro del marco de muestreo $\left(1 \mathrm{~m}^{2}\right)$ y tomando en cuenta la escala que se muestra en la Figura 2.

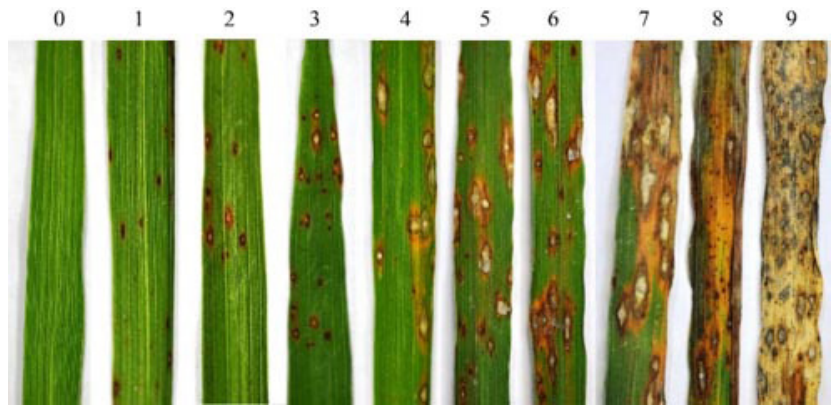

Figura 2. Escala de severidad (0-9) para manchas foliares causadas por Pyricularia grisea en raigrás (Lolium sp.) (ETHzürich, 2016).

Figure 2. Scale for leaf spots severity (0-9) caused by Pyricularia grisea on Ryegrass (Lolium sp.) (ETHzürich, 2016).

\section{Rendimiento de materia seca}

Durante la fase productiva, después del corte de uniformización (90 días después de la siembra), se realizaron ciclos de corte en cada localidad: en Pasto, dos en época de lluvias y dos en época seca; en Sapuyes, uno en lluviosa y tres en seca, y en Cumbal, uno en lluviosa y dos en seca. Para determinar el rendimiento de forraje verde, en cada ciclo se realizaron cosechas o cortes a $\operatorname{los} 25,30,35$ y 40 días, cortando el forraje presente dentro del marco de muestreo $\left(1 \mathrm{~m}^{2}\right)$ a $5 \mathrm{~cm}$ de altura sobre el suelo. De cada muestra de forraje verde, se tomó una submuestra de $500 \mathrm{~g}$ y en bolsas de papel se colocaron en horno de secado a temperatura de $70{ }^{\circ} \mathrm{C}$ durante $48 \mathrm{~h}$ (CIAT, 1982).

\section{Resultados}

\section{Altura de planta}

La altura de planta estuvo afectada en forma altamente significativa $(\mathrm{p}<0,01)$ por los factores genotipo, localidad y edad de cosecha, así como por las interacciones genotipo x localidad, genotipo x época, genotipo $\mathrm{x}$ edad de cosecha, genotipo x localidad x época además, el factor época la afectó de forma significativa $(\mathrm{p}<0,05)$.

Se encontró para los genotipos y la edad de cosecha que, la mayor altura $(\mathrm{cm})$ a los 25 días la presentó Angus $(25,10)$; a los 30,35 y 40 días Conquistador $(32,33,37,79$ y 42,07 , respectivamente), pero Angus $(42,02)$ también fue de los más altos a los 40 días. Teniendo en cuenta la interacción genotipo x localidad x época, Conquistador $(46,33)$ presentó la mayor altura en la localidad de Pasto en época lluviosa; en esta misma época se destacó Bestfor Plus con 32,60 cm en Cumbal y con 25,33 cm en Sapuyes. En la época seca, el genotipo con mayor altura (cm) fue Magnum $(40,72)$ en la localidad de Pasto y Conquistador en Cumbal $(31,95)$ y Sapuyes $(32,64)$ (Figura 3$)$. 

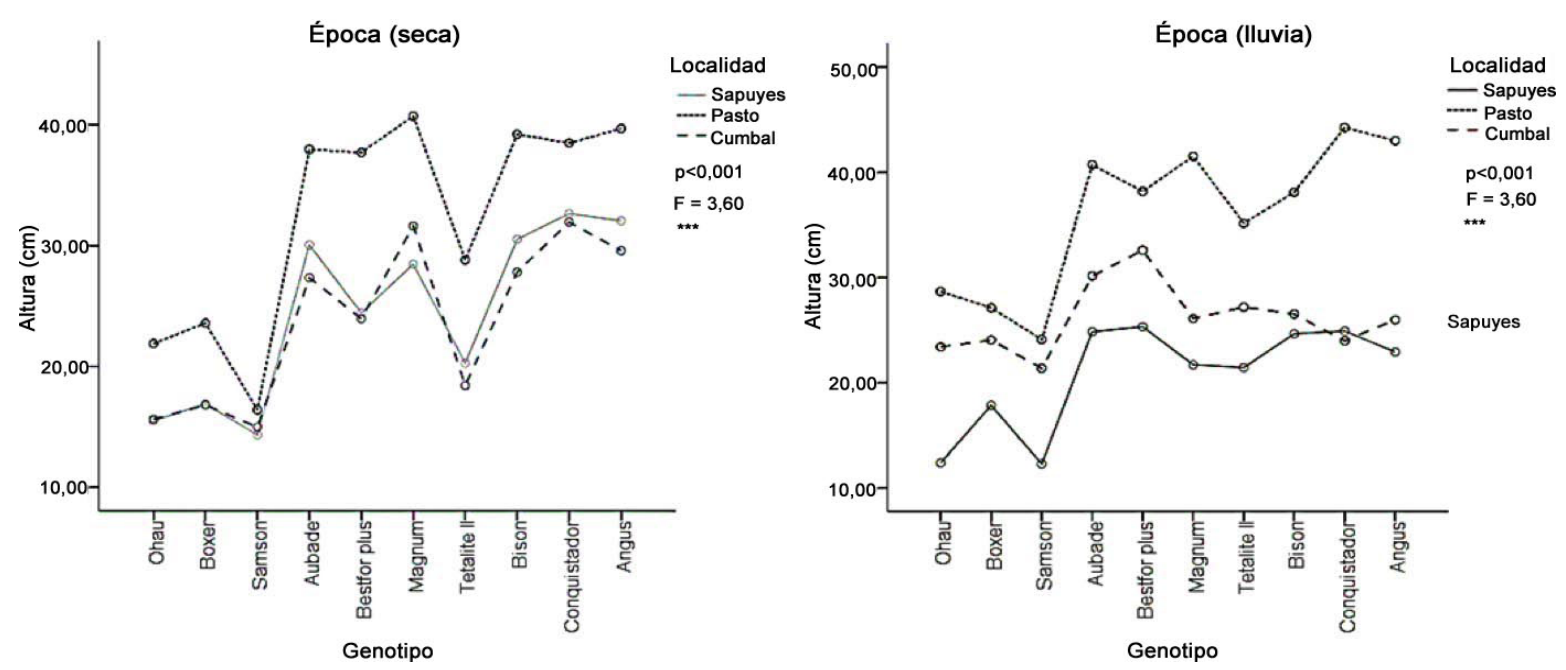

Figura 3. Interacción genotipo x localidad x época para la variable altura de planta $(\mathrm{cm})$ en diez genotipos de raigrás (Lolium sp.), evaluados en tres localidades de la zona alta de Nariño, Colombia. 2016-2017.

Figure 3. Genotype $\mathrm{x}$ locality $\mathrm{x}$ season interaction for height of the plant $(\mathrm{cm})$ variable in ten Ryegrass (Lolium sp.) genotypes evaluated in three localities of the high lands of Nariño, Colombia. 2016-2017.

\section{Cobertura}

En cobertura se encontraron efectos altamente significativos de los factores genotipo, localidad, época y de las interacciones genotipo $\mathrm{x}$ localidad, genotipo x época de cosecha y genotipo x localidad x época.

En la interacción genotipo x localidad x época, los genotipos Aubade $(99,67)$ y Angus $(99,64)$ presentaron la mayor cobertura (\%) en la localidad de Pasto en época lluviosa, en esta misma época, Aubade $(90,62)$ también fue el de mayor cobertura en Cumbal y Boxer $(83,50)$ en Sapuyes. En época seca, el genotipo de mayor cobertura fue Samson $(98,53)$ en Pasto, en Cumbal fue Aubade $(76,78)$ y en Sapuyes Boxer $(76,87)$ (Figura 4).

\section{Severidad de enfermedades foliares}

La severidad de las enfermedades foliares estuvo influida en forma altamente significativa por genotipo, época, edad de cosecha y por las interacciones genotipo x localidad, genotipo x edad de cosecha, genotipo x localidad $\mathrm{x}$ época y genotipo x localidad $\mathrm{x}$ edad de cosecha, así como en forma significativa por el factor localidades.

Los genotipos de raigrás estuvieron poco afectados por enfermedades, sin embargo, la que se presentó con mayor frecuencia fue la mancha foliar causada por Pyricularia spp., que generó lesiones necróticas en el follaje de los pastos.

Los genotipos Samson $(1,00)$, Conquistador $(1,00)$ y Angus $(1,00)$ presentaron la severidad más baja en la localidad de Sapuyes en época lluviosa. En esta misma época y en Cumbal sobresalió Boxer $(1,06)$, y en Pasto el genotipo Samson $(1,14)$. En época seca también se destacó el genotipo Samson, por presentar los valores más bajos de severidad en Cumbal $(1,12)$, en Sapuyes $(1,14)$ y en Pasto $(1,18)$ (Figura 5).

Con base en la interacción genotipo x localidad x edad de cosecha, los genotipos Samson, Ohau y Boxer, en Cumbal y a los 25 días, presentaron la severidad más baja, mientras que a los 30, 35 y 40 días fue Samson (con 1,$16 ; 1,33$ y 1,25 respectivamente). Este mismo genotipo se comportó de manera similar en Sapuyes a los $25(1,0)$, $30(1,06), 35(1,0)$ y 40 días $(1,37)$, y de la misma forma en Pasto (con valores entre 1,12 y 1,19) (Figura 6 ). 

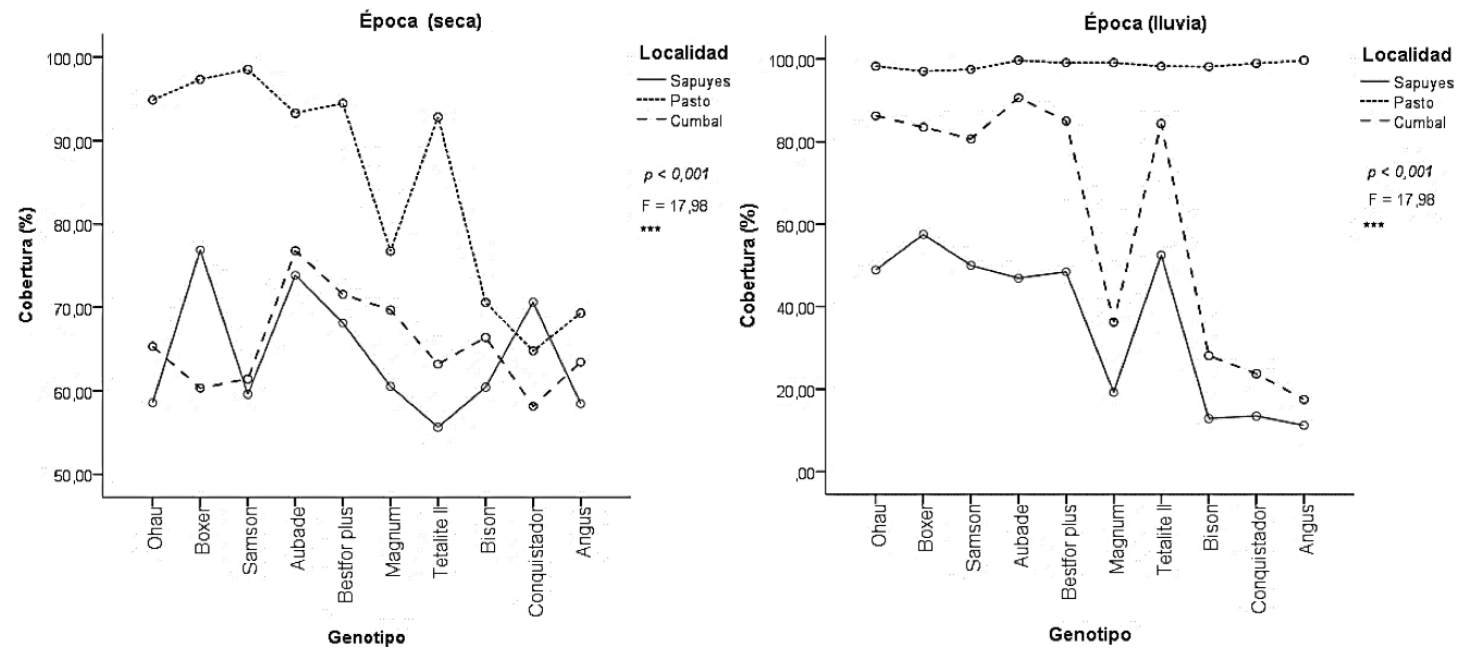

Figura 4. Interacción genotipo $\mathrm{x}$ localidad $\mathrm{x}$ época para la variable cobertura (\%) en diez genotipos de raigrás (Lolium sp.), evaluados en tres localidades de la zona alta de Nariño, Colombia. 2016-2017.

Figure 4. Genotype $\mathrm{x}$ locality $\mathrm{x}$ season interaction for the coverage (\%) variable for ten Ryegrass (Lolium sp.) genotypes evaluated in three localities of the high lands of Nariño, Colombia during dry and rainy seasons. 2016-2017.
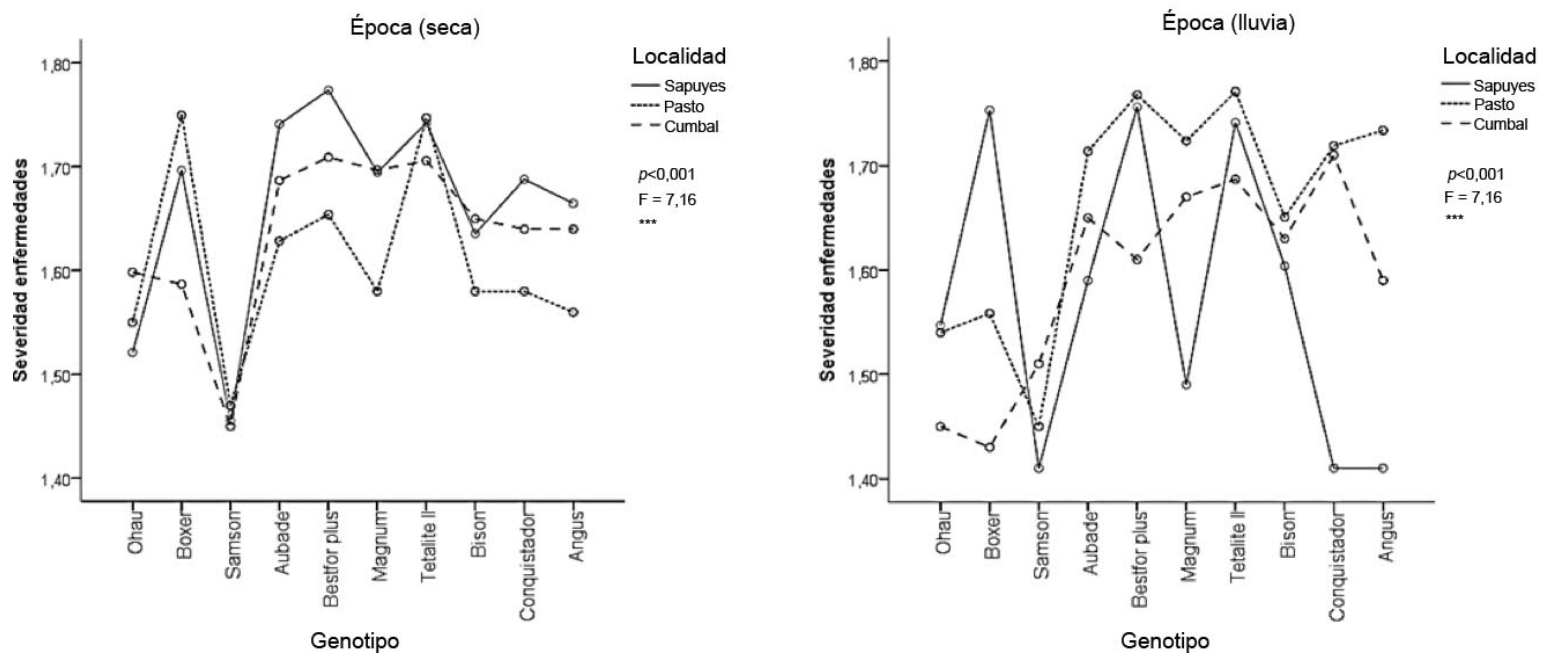

Figura 5. Interacción genotipo $\mathrm{x}$ localidad $\mathrm{x}$ época para la variable severidad de enfermedades en diez genotipos de raigrás (Lolium sp.), evaluados en tres localidades de la zona alta de Nariño, Colombia. 2016-2017.

Figure 5. Genotype $x$ locality $x$ season interaction for the severity of diseases variable for ten Ryegrass (Lolium sp.) genotypes evaluated in three localities of the high lands of Nariño, Colombia during dry and rainy seasons. 2016-2017.

\section{Rendimiento de materia seca}

El rendimiento de materia seca $(\mathrm{t} / \mathrm{ha} /$ corte $)$ estuvo afectado $(\mathrm{p}<0,01)$ por los factores genotipo, localidad, época, edad de cosecha y por las interacciones genotipo x localidad, genotipo x época y genotipo x localidad x época. 

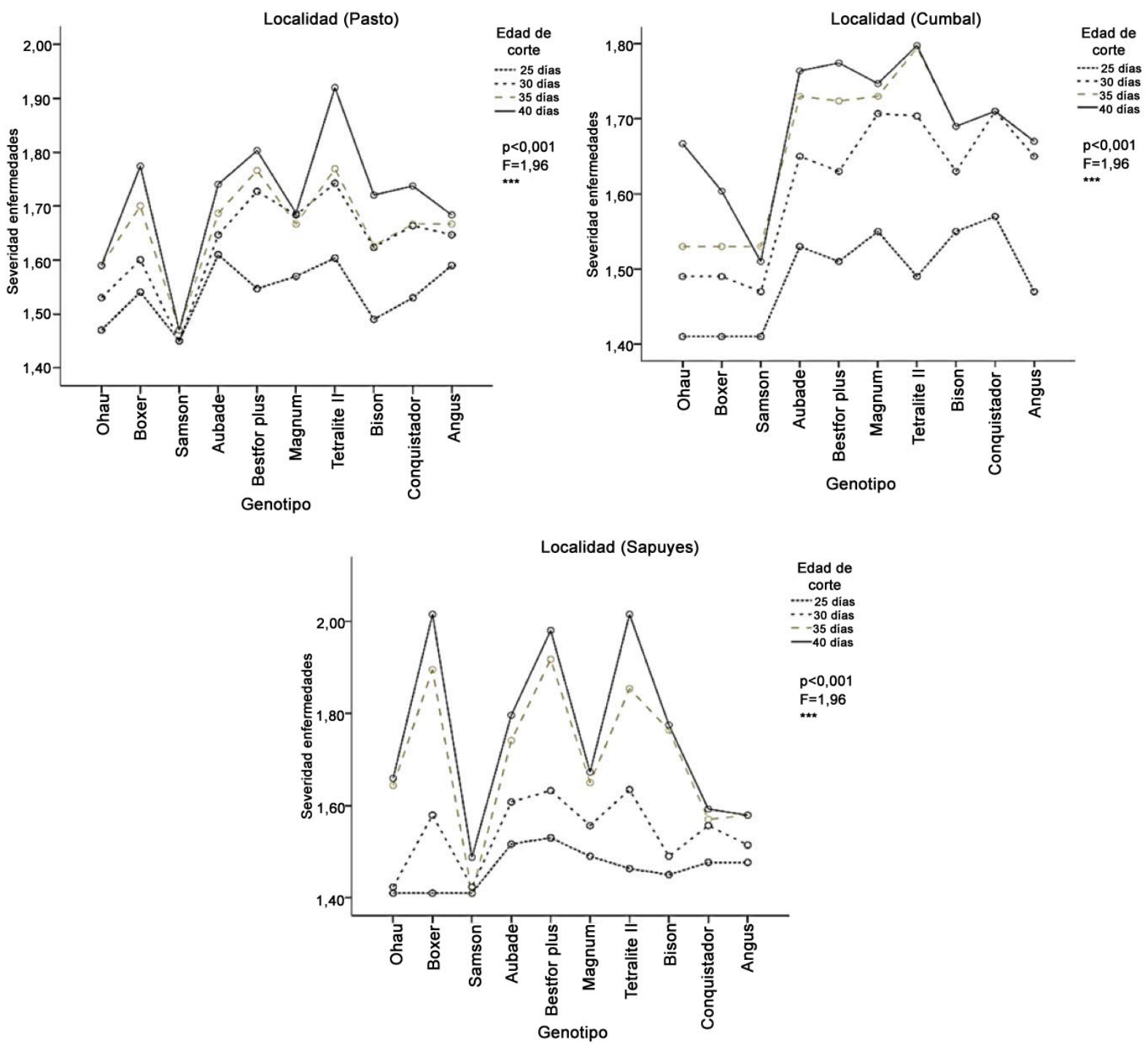

Figura 6. Interacción genotipo $\mathrm{x}$ localidad $\mathrm{x}$ edad de cosecha para la variable severidad de enfermedades en diez genotipos de raigrás (Lolium sp.), evaluados en tres localidades de la zona alta de Nariño, Colombia. 2016-2017.

Figure 6. Genotype $\mathrm{x}$ locality $\mathrm{x}$ time of harvesting interaction for the severity of diseases variable for ten Ryegrass (Lolium sp.) genotypes evaluated in three localities of the high lands of Nariño, Colombia during dry and rainy seasons. 2016-2017.

Los genotipos con mejor rendimiento de materia seca ( $\mathrm{t} / \mathrm{ha} / \mathrm{corte}$ ) en la localidad de Pasto y en época de lluvias fueron: Tetralite II $(2,22)$, Bestfor Plus $(2,17)$, Aubade $(2,11)$ y Boxer $(2,09)$, en esta misma época Bestfor Plus $(1,60)$ y Tetralite II $(1,59)$ fueron los de mejor comportamiento en Cumbal y también en Sapuyes (con 0,66 y 0,65 , respectivamente). En época seca los rendimientos más altos los obtuvieron los genotipos Boxer $(2,10)$, Tetralite II $(1,97)$ y Bestfor Plus $(1,93)$ en Pasto, Magnum $(1,15)$, Aubade $(1,10)$ y Bestfor Plus $(1,09)$ en Cumbal, y Conquistador $(1,05)$ en Sapuyes (Figura 7). 

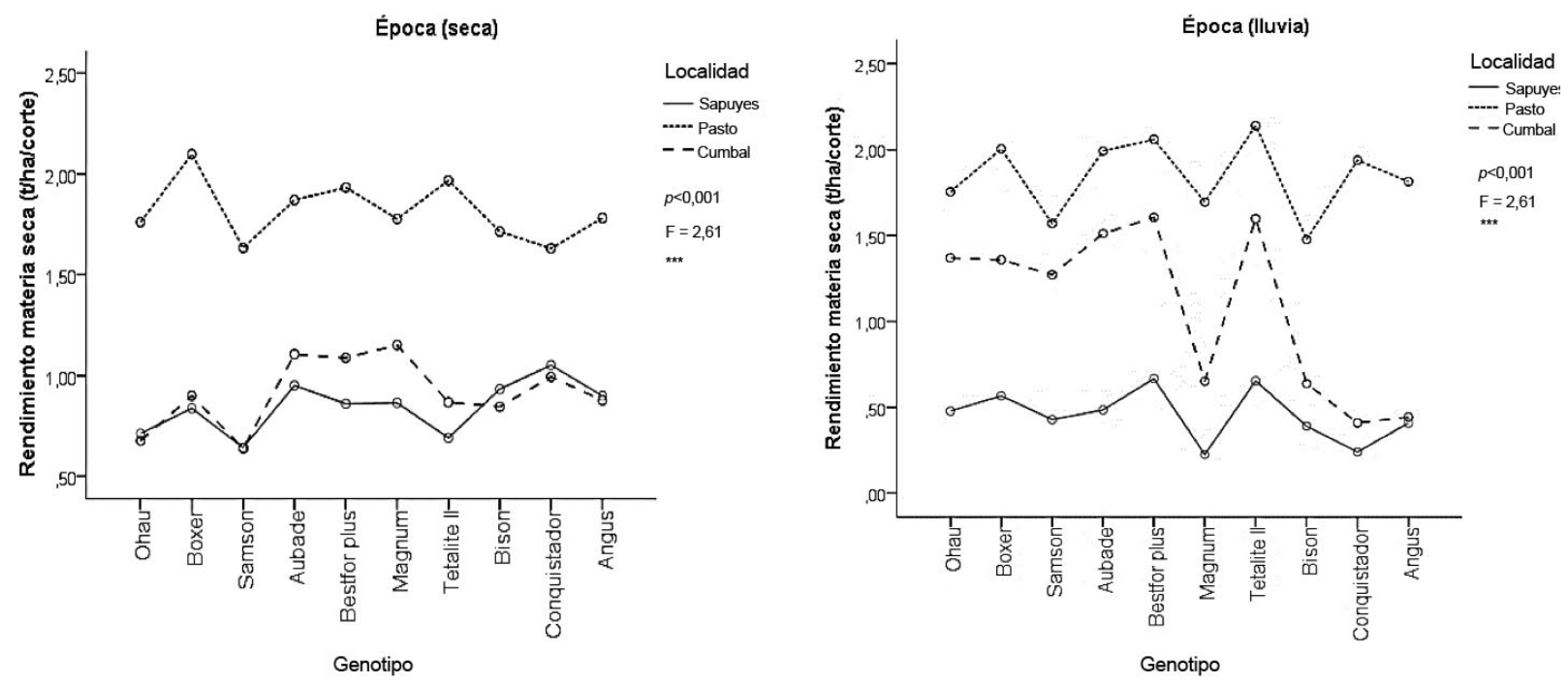

Figura 7. Interacción genotipo $x$ localidad $x$ edad de cosecha para la variable rendimiento de materia seca (t/ha/corte) en diez genotipos de raigrás (Lolium sp.) evaluados en tres localidades de la zona alta de Nariño, Colombia. 2016-2017.

Figure 7. Genotype $x$ location $x$ harvest age interaction for the yield of dry matter ( $t / h a / c u t)$ variable in ten genotypes of ryegrass (Lolium sp.) evaluated in three locations of the high lands of Nariño, Colombia. 2016-2017.

\section{Discusión}

\section{Altura de planta}

Los mayores valores de altura de planta $(\mathrm{cm})$ se observaron en la localidad de Pasto, debido en gran parte a que en esta localidad hubo una precipitación más alta que en Cumbal y Sapuyes. Además, la altura de los raigrases fue mayor en época lluviosa, lo cual es coherente, dado que, el crecimiento de las plantas forrajeras gramíneas está influenciado por condiciones ambientales (Aguirre, 2013) y el agua es uno de los factores climáticos que afectan en gran medida en el crecimiento del raigrás, por tanto, los resultados obtenidos se atribuyen al déficit hídrico en la época seca, el cual influyó en el crecimiento y tamaño final de las hojas (Kramer, 1983; Nuñez-Barrios y Foster, 1996).

La edad de cosecha afectó la altura de los genotipos, a los 40 días se logró el mayor crecimiento de estos, debido al tiempo transcurrido entre el corte de uniformización y el siguiente corte; el paso de un mayor número de días permitió un mayor crecimiento de las plantas (Acharán et al., 2009). En un estudio con Lolium perenne, también se encontró que entre los 28 y 42 días se obtuvieron los mayores valores de altura (Velasco et al., 2005). Los genotipos más altos (Conquistador y Angus) correspondieron a raigrases tipo híbridos y anuales, que se caracterizaron por presentar alturas superiores a los perennes. Tambien se encontró en un raigrás híbrido y dos anuales (Posada et al., 2013), valores de altura superiores a $30 \mathrm{~cm}$ cuando se realizaron cortes a los 35 días. Según esta investigación, la altura de las plantas es un factor que favorece la mayor permanencia de las especies cultivadas en el potrero, ya que el animal no agotará las reservas necesarias para la recuperación de la pradera.

Las concentraciones de alcaloides en raigrás, producidas por hongos endófitos, aumentan dependiendo del genotipo, la edad y la estación (verano), los cuales le confieren una defensa adicional a las pasturas contra plagas, patógenos y herbívoros, lo que provoca un mayor crecimiento (Fuchs et al., 2017), y coincide con lo encontrado en 
este estudio, donde al observar la interacción entre estos factores, los raigrases, Magnum y Conquistador alcanzaron las mayores alturas, debido a su condición híbrida y tetraploide.

\section{Cobertura}

El valor más alto de cobertura (\%) se alcanzó en Pasto, debido a las condiciones edafoclimáticas de esta localidad, principalmente el agua de lluvia que fue mayor, lo que favoreció el adecuado crecimiento y desarrollo de estas gramíneas.

El porcentaje de cobertura más alto se encontró en la época lluviosa. Esto pudo ocurrir debido a que, en periodos lluviosos, la disponibilidad de nutrientes se hace mayor, permitiendo un desarrollo normal de las plantas; mientras que, en periodos secos si las especies no son tolerantes, la producción de forraje disminuye y aumenta la invasión de malezas (Franco et al., 2005), lo que repercute en bajos porcentajes de cobertura de la especie de interés.

\section{Severidad de enfermedades foliares}

En general, los valores de severidad de Pyricularia spp. encontrados en este estudio fueron relativamente bajos, al tener como valor máximo el grado 2 (11-20\% de infección). En el corte a los 40 días se presentó la mayor severidad de esta enfermedad, debido a que en esta investigación no se realizó ningún control químico de la enfermedad, estos resultados son normales, considerando que, a medida que pasa el tiempo, y si no hay un manejo de la enfermedad, la severidad irá en aumento. Samson fue el genotipo menos afectado (menos del 10\% de infección), el cual al demostrar mayor grado de tolerancia a Pyricularia spp. podría tenerse en cuenta para futuros estudios de resistencia y/o tolerancia a enfermedades.

Se ha propuesto que además de producir alcaloides protectores, los hongos endófitos asociados a raigrases mejoran la inmunidad de las plantas contra enfermedades, por la promoción de endógenas mediadas por la vía del ácido jasmónico (JA) (Bastias et al., 2017), esto podría explicar lo encontrado en el presente estudio, donde los niveles de severidad en general, no superaron el 20\%, además concuerda con lo expresado por los mismos autores donde se expone que diferentes escenarios ecológicos inciden sobre el efecto del endófito en las defensas de las plantas, aumentando así la aptitud de la planta, lo que muestra que en este estudio las localidades de Pasto y Cumbal presentaron las mejores condiciones para el desarrollo de estas características en los diferentes genotipos de raigrás evaluados.

\section{Rendimiento de materia seca}

En general, se pudo apreciar que los mayores rendimientos de materia seca (t/ha/corte) se alcanzaron con los genotipos evaluados en la localidad de Pasto, seguidos de Cumbal y Sapuyes; esto debido en gran parte a las diferentes condiciones agroclimáticas de cada localidad, en donde Pasto se caracterizó por presentar condiciones más favorables, en temperatura, precipitación y suelo, permitiendo un adecuado crecimiento, desarrollo y producción de los raigrases. Cuando se evaluaron cinco variedades de raigrás en dos localidades (Vargas et al., 2018), se obtuvieron rendimientos superiores a los encontrados en la presente investigación en la localidad de Tuta ( $2,51 \mathrm{t} / \mathrm{ha} /$ corte), pero muy inferiores en Mosquera $(0,47 \mathrm{t} / \mathrm{ha} /$ corte $)$, indicando una respuesta diferencial de los genotipos, debido posiblemente a las características del ambiente de evaluación.

Los resultados encontrados en la interacción genotipo x localidad indican la gran capacidad de producción de los genotipos al ser evaluados en Pasto, si se consideran nueve cortes al año, los diez genotipos presentaron 
rendimientos superiores a 14,60 t/ha/año, con un 21,6\% de incremento sobre el promedio de las zonas altas de Colombia que corresponde a $12 \mathrm{t} / \mathrm{ha} /$ año (SENA, 1985).

Los mejores rendimientos de materia seca ( $\mathrm{t} / \mathrm{ha} /$ corte) de los genotipos se presentaron en época lluviosa, lo cual concuerda con lo mencionado por Villalobos y Sánchez (2010), quienes observaron que, "las características ambientales influyen sobre la producción de materia seca de los pastos”. Además, Álvarez et al. (2012) observaron que los pastos presentan altas producciones durante el periodo lluvioso y disminuyen en la época de pocas precipitaciones; lo que reiteran Ramírez-de-la-Ribera et al. (2017) y Sosa et al. (2008), cuando mencionan que los pastos en general, reducen su rendimiento en condiciones de poca humedad o precipitaciones. Se indicó que "las lluvias y la temperatura, a lo largo del año, son los principales elementos del clima que determinan la adaptación e influyen en el rendimiento de las especies forrajeras" (Chaput, 2015), y la mayor productividad de los pastos se presenta en la época de lluvias (Cuadrado et al., 2003; Bernal, 2005).

En un déficit severo de agua en el suelo, el raigrás desarrolla un sistema radical más grande y aumenta su capacidad para controlar las pérdidas por transpiración de manera más eficiente, lo que prolonga su período de crecimiento y se ve reflejado en los rendimientos de materia seca, coincidiendo con lo encontrado en este estudio, ya que a pesar de disminuir las precipitaciones en época seca los genotipos con características de tetraploides presentaron rendimientos similares a las encontradas en la época de lluvias (Lucero et al., 1999).

A los 40 días se presentaron los rendimientos más altos. Resultados similares encontraron Velasco et al. (2005) en un estudio realizado en México, para determinar la frecuencia de corte que proporcione mayor rendimiento de materia seca y valor nutritivo en ballico perenne (Lolium perenne L.), en donde encontraron que la mayor acumulación de materia seca (6,00 t/ha/año) se obtiene al cosechar entre la semana 4 y 6 ( 28 y 42 días). En otro estudio en México, al evaluar la influencia de la edad de cosecha de Pennisetum purpureum, con el aumento de la edad de corte se incrementó el rendimiento de materia seca (Madera et al., 2013). Lo anterior concuerda con lo mencionado en la revisión bibliográfica de Lescano (2009), donde se afirma que el momento óptimo de defoliación de algunas especies de Lolium para obtener la máxima producción de forraje debe ser entre la $5^{\circ}$ y $6^{\circ}$ semana (3542 días).

Los rendimientos obtenidos en esta investigación, al ser comparados con los del pasto kikuyo, gramínea predominante en la zona alta de Nariño, fueron inferiores según los reportes de varios estudios (León y Zambrano, 2008; Gómez et al., 2013), pero cabe resaltar que esta es una especie que no está recomendada para zonas que presentan heladas durante el año, como es el caso de las localidades de este estudio. Según Benavides (2009), las plantas no son capaces de mantener su temperatura constante, por lo que, los cambios de temperatura ambiental influyen sobre su crecimiento y desarrollo. El kikuyo es una especie tolerante a sequías, pero muy susceptible a las heladas (Cárdenas, 2008; Castro et al., 2009); mientras que, el raigrás es una especie que tolera temperaturas bajas que se presentan en algunas zonas (Villalobos y Sánchez, 2010).

\section{Conclusiones}

Los genotipos Tetralite II, Besfort Plus y Boxer presentaron buena adaptación en los tres ambientes de evaluación (Pasto, Cumbal y Sapuyes), por lo que representan una opción para diversificar la oferta forrajera en estas localidades. Se sugiere su validación en el sector ganadero de las zonas altas de Nariño.

La respuesta de los genotipos frente a las épocas seca y lluviosa fue marcada, lo que favoreció el rendimiento de materia seca, altura de planta y cobertura durante la época lluviosa.

La evaluación de adaptación de los raigrases fue de gran importancia para determinar su comportamiento agronómico en los tres ambientes, sin embargo, es necesario complementar este estudio con evaluaciones en otras localidades de las zonas altas de Colombia y considerar también variables de calidad nutricional (proteína, fibra detergente neutro, fibra detergente ácida y digestibilidad) y evaluación en un modelo animal. 


\section{Agradecimientos}

Los autores expresan sus agradecimientos a la Corporación Colombiana de Investigación Agropecuaria (AGROSAVIA), a la Sociedad de Agricultores y Ganaderos de Nariño (SAGAN) y a la Universidad de Nariño por haber permitido llevar a cabo esta investigación a través del Macroproyecto denominado "Mejoramiento de la oferta forrajera, optimización de sistemas de alimentación y aseguramiento de la calidad e inocuidad de la leche en el trópico alto del departamento de Nariño" con recursos del Sistema General de Regalías.

\section{Literatura citada}

Acharán, F., O. Balocchi, y I. López. 2009. Filocrono, producción de fitomasa y calidad nutritiva de una pradera de Lolium perenne L./Trifolium repens L. sometida a tres frecuencias e intensidades de defoliación. Agro Sur 37(2):81-90. doi:10.4206/agrosur. 2009.v37n2-02.

AGROSAVIA (Corporación Colombiana de Investigación Agropecuaria). 2016. Mejoramiento de la oferta forrajera, optimización de sistemas de alimentación y aseguramiento de la calidad e inocuidad de la leche en el trópico alto del departamento de Nariño. AGROSAVIA, Bogotá, COL.

Aguirre, L. 2013. Establecimiento y manejo de praderas según procedimiento. En: J.A. Leal et al., editores, Módulo curso: Prácticas básicas para la producción de forrajes. Universidad Nacional Abierta y a Distancia, Bogotá, COL. p. 4-93.

Álvarez, A., R. Herrera, A. Noda, y L. Díaz. 2012. Comportamiento de las precipitaciones en el Instituto de Ciencia Animal en Cuba durante el período 1970-2009, como base para el manejo estratégico de los pastos. Rev. Cubana Cienc. Agric. 46:301-307.

Bastias, D.A., M.A. Martínez-Ghersa, C.L. Ballaré, and P.E. Gunde. 2017. Epichloë fungal endophytes and plant defenses: not just alkaloids. Trends Plant Sci. 22:939-948 doi:10.1016/j.tplants.2017.08.005

Benavides, A. 2009. Temas modernos de nutrición vegetal. Sociedad Mexicana de la Ciencia del Suelo, A.C., Saltillo, MEX.

Bernal, J. 2005. Manual de manejo de pastos cultivados para zonas altoandinas. Dirección General de Producción Agraria (DGPA), PER.

Cárdenas, E.A. 2008. Alternativas forrajeras para clima frío en Colombia. Scribd. https://es.scribd.com/doc/74111208/ gramineas-climas-frios (consultado 18 set. 2018).

Castro, E., J.E. Mojica, J. León, M. Pabón, J. Carulla, y E. Cárdenas. 2009. Balance de nitrógeno en pastura de gramínea más Lotus uliginosus en la sabana de Bogotá, Colombia. Rev Cienc. Tecnol. Agropecu. 10:91-101. doi:10.21930/rcta.vol10num 1

Castrillón, D. 2014. Cuencas lecheras, motores de la producción nacional. Informe. Contexto ganadero. CONtextoganadero, COL. http://www.contextoganadero.com/ganaderia-sostenible/informe-cuencas-lecheras-motores-de-la-produccionnacional (consultado 07 abr. 2018).

Chaput, P. (ed.). 2015. Programa de gestión rural empresarial, sanidad y ambiente: Pastos y forrajes. Catholic Relief Services (CRS), Managua, NIC.

CIAT. 1982. Manual para la evaluación agronómica. CIAT, Cali, COL.

Climte-Data.Org. 2018. Clima: Pasto, Cumbal y Sapuyes. Climte-Data.Org. https://es.climate-data.org/location/3811/ (consultado 15 mar. 2018). 
Cuadrado, H., S. Mejía, A. Contreras, A. Romero, y J. García. 2003. Manejo agronómico de algunos cultivos forrajeros y técnicas para su conservación en la región caribe colombiano. Manual técnico. Corpoica, Centro de investigación Turipaná. Córdoba, COL.

Cuesta, P.A. 2008. Evaluación y selección de nuevas especies forrajeras para mejorar la eficiencia de los sistemas ganaderos de leche de trópico alto colombiano. Produmedios, Bogotá, COL.

ETHzürich. 2016. Identification of novel rice blast resistance sources. Institute of Molecular Plant Biology, SUI. http://www. impb.ethz.ch/research/reseach-pb/research-pb/group_pb_cereal/resistancessources.html (accessed 02 Feb., 2016).

FAO. 2018. Producción animal: El papel de la FAO en la producción animal. FAO, Roma, ITA. http://www.fao.org/animalproduction/es/ (consultado 18 set. 2018).

Franco, L.H., D. Calero, y C.V. Durán. 2005. Manejo y utilización de forrajes tropicales multipropósito. Proyecto: Evaluación de tecnologías por métodos participativos para la implementación de sistemas ganaderos sostenibles en el norte del departamento del Valle del Cauca. CIAT, y Universidad Nacional de Colombia, COL.

Fuchs, B., M. Krischke, M.J. Mueller, and J. Krauss. 2017. Plant age and seasonal timing determine endophyte growth and alkaloid biosynthesis. Fungal Ecol. 29:52-58 doi:10.1016/j.funeco.2017.06.003

Gómez A., A. Silva, J. Salazar, y J. Andrade. 2013. Producción de materia seca y calidad del pasto kikuyo P. clandestinum en diferentes niveles de fertilización nitrogenada y en asocio con aliso (Alnus acuminata) en el trópico alto colombiano. En: V. Porfírio-da-Silva, editor, Anais do $1^{\circ}$ Simpósio Internacional de Arborização de Pastagens em Regiões Subtropicais. Embrapa Florestas, BRA. p. 32-41.

Kramer, P. 1983. Water relations of plants. Academic Press Inc, N.Y. USA.

León, J., y D. Zambrano. 2008. Determinación de los factores edafoclimáticos que intervienen en la producción y calidad nutritiva del pasto kikuyo (Pennisetum clandestinum Hoeschst) en condiciones de no intervención en el municipio de Pasto, Departamento de Nariño. Tesis Lic., Universidad de Nariño, COL.

Lescano, F. 2009. Establecimiento de la asociación Rye grass (Lolium multiflorum) Trébol Blanco (Trifolium repens). Universidad Nacional de Cajamarca. PER.

Lucero, D.W., P. Grieu, and A. Guckert. 1999. Effects of water deficit and plant interaction on morphological growth parameters and yield of white clover (Trifolium repens L.) and ryegrass (Lolium perenne L.) mixture. Eur. J. Agron. 11(3-4):167-177 doi:10.1016/S1161-0301(99)00028-3

Madera, N., B. Ortiz, H. Bacab, y H. Magaña. 2013. Influencia de la edad de corte del pasto morado (Pennisetum purpureum) en la producción y digestibilidad in vitro de la materia seca. Avanc. Investig. Agropec. 17(2):41-52.

MADR (Ministerio de Agricultura y Desarrollo Rural) y MinCIT (Ministerio de Comercio, Industria y Turismo). 2016. Implementación política para mejorar la competitividad del sector lácteo nacional. Programa de apoyo presupuestario al sector lácteo en Colombia en desarrollo del programa DCI-ALA/2012/024-513. MADR, COL. https://www. minagricultura.gov.co/ministerio/direcciones/Documents/INFORME\%20UE\%20FASE\%202\%20-SEGUNDO\%20 TRAMO\%20VARIABLE\%20\%28MARZO\%202016\%29.pdf (consultado 09 may. 2018).

Nuñez-Barrios, A., y E. Foster. 1996. Efecto del déficit hídrico sobre el crecimiento de hojas, tallos y vainas de frijol. Agric. Téc. Méx. 22(1):99-109.

Pinto, A. 2017. Sector lechero en Colombia: Potencial desperdiciado. Universidad de los Andes. Bogotá, COL. https:// agronegocios.uniandes.edu.co/2017/09/22/sector-lechero-en-colombia-potencial-desperdiciado/ (consultado 07 abr. 2018). 
Posada, S., J.M. Cerón, J. Arenas, J.F. Hamedt, y A. Álvarez. 2013. Evaluación del establecimiento de raigrás (Lolium sp.) en potreros de kikuyo (Pennisetum clandestinum) usando la metodología de cero labranza. CES Med. Vet. Zootec. 8(1):2635.

Ramírez-de-la-Ribera, J., D. Zambrano-Burgos, J. Campuzano, D. Verdecia-Acosta, E. Chacón-Marcheco, Y. Arceo-Benítez, J. Labrada-Ching, y H. Uvidia-Cabadiana. 2017. El clima y su influencia en la producción de los pastos. REDVET. 18(6). http://www.veterinaria.org/revistas/redvet/n060617/061701.pdf (consultado 19 jul. 2018).

Secretaria de Agricultura y Desarrollo Rural de Nariño, y Universidad Sergio Arboleda. 2016. Consolidado agropecuario de Nariño 2016. Secretaria de Agricultura y Desarrollo Rural de Nariño, y Universidad Sergio Arboleda, Nariño, COL.

SENA (Servicio Nacional de Aprendizaje). 1985. Cartilla 3: Pastos y forrajes de clima frío. Servicio Nacional de Aprendizaje, Bogotá, COL.

Sosa-Rubio, E., E. Cabrera-Torres, D. Pérez-Rodríguez, y L. Ortega-Reyes. 2008. Producción estacional de materia seca de gramíneas y leguminosas forrajeras con cortes en el estado de Quintana Roo. Téc. Pecu. Méx. 46:413-426.

Vargas, J., A. Sierra, J. Benavides, Y. Avellaneda, O. Mayorga, y C. Ariza. 2018. Establecimiento y producción de raigrás y tréboles en dos regiones del trópico alto colombiano. Agron. Mesoam. 29:177-191. doi:10.15517/ma.v29i1.28077

Velasco, M., A. Hernández, y V. Gonzáles. 2005. Rendimiento y valor nutritivo del ballico perenne (Lolium perenne L.) en respuesta a la frecuencia de corte. Téc. Pecu. Méx. 43(2):247-258.

Villalobos, L., y J. Sánchez. 2010. Evaluación agronómica y nutricional del pasto raigrás perenne tetraploide (Lolium perenne) producido en lecherías de las zonas altas de Costa Rica. I. Producción de biomasa y fenología. Agron. Costarr. 34:31-42. 